


\section{The Nerve/Tunnel Index: A New Diagnostic Standard for Carpal Tunnel Syndrome Using Sonography}

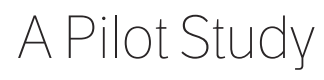

Hyoung Seop Kim, MD, Seung Ho Joo, MD, Zee-A Han, MD, Yong Wook Kim, MD, PhD

Received March 25, 2011, from the Departments of Physical Medicine and Rehabilitation (H.S.K., Z.H.) and Diagnostic Radiology (S.H.J.), National Health Insurance Corporation Ilsan Hospital, Gyonggi-Do, Korea; and Department and Research Institute of Rehabilitation Medicine, Yonsei University College of Medicine Severance Hospital, Seoul, Korea (Y.W.K.). Revision requested April 18, 2011. Revised manuscript accepted for publication August 4, 2011.

We thank Jong Moon Kim, MD, and Jin Young Park, MD, for data collection and management and Jung Bin Shin, $M D$, and Seong Woo Kim, MD, for suggestions and assistance.

Address correspondence to Yong Wook Kim, $M D, P h D$, Department of Rehabilitation Medicine, Yonsei University College of Medicine, 250 Seongsanno, Seodaemun-Gu, Seoul 120-752, Korea.

E-mail:ywkim1@yuhs.ac

Abbreviations

BMI, body mass index
Objectives - To define the relationship between body indices of healthy adults and cross-sectional areas of the carpal tunnel and median nerve and to obtain the nerve/tunnel index, which represents a new standard for diagnosing carpal tunnel syndrome using sonography.

Methods-Body indices (height, weight, and body mass index) were analyzed in 60 healthy adults, and electromyography and sonography were also performed. The crosssectional areas of the proximal and distal median nerve and carpal tunnel were obtained by sonography. The proximal and distal nerve/tunnel indices were obtained by calculating the ratio between the proximal and distal cross-sectional areas of the median nerve to those of the carpal tunnel and multiplying the value by 100 .

Results-Although the proximal cross-sectional areas of the median nerve and body indices showed statistically significant relationships with weak positive correlations, the proximal and distal areas of the carpal tunnel showed relatively stronger correlations with body indices. Between sexes, there were significant differences in the proximal median nerve cross-sectional area (mean \pm SD: male, $10.48 \pm 3.21 \mathrm{~mm}^{2}$; female, $8.81 \pm$ $3.21 \mathrm{~mm}^{2} ; P<.05$ ) and proximal carpal tunnel area (male, $182.50 \pm 21.15 \mathrm{~mm}^{2}$; female, $\left.151.23 \pm 21.14 \mathrm{~mm}^{2} ; P<.05\right)$. There was no difference in the proximal nerve/tunnel index (male, $5.80 \% \pm 1.72 \%$; female, $5.91 \% \pm 1.63 \%$ ). There was a statistically significant difference in the distal carpal tunnel cross-sectional area (male, 138.90 $\pm 20.95 \mathrm{~mm}^{2}$; female, $121.50 \pm 18.99 \mathrm{~mm}^{2} ; P<.05$ ) between sexes, but the distal median area (male, $9.99 \pm 3.42 \mathrm{~mm}^{2}$; female, $8.46 \pm 1.84 \mathrm{~mm}^{2}$ ) and distal nerve/tunnel index (male, $7.15 \% \pm 2.00 \%$; female, $7.01 \% \pm 1.38 \%$ ) showed no significant differences. The proximal index was significantly higher than the distal index (proximal, $5.85 \% \pm$ $1.66 \%$; distal, $7.08 \% \pm 1.71 \%)$.

Conclusions - The nerve/tunnel index is unaffected by body indices or sex and thus may be a useful and objective standard for diagnosing carpal tunnel syndrome.

Key Words — carpal tunnel syndrome; median nerve; nerve/tunnel index; sonography

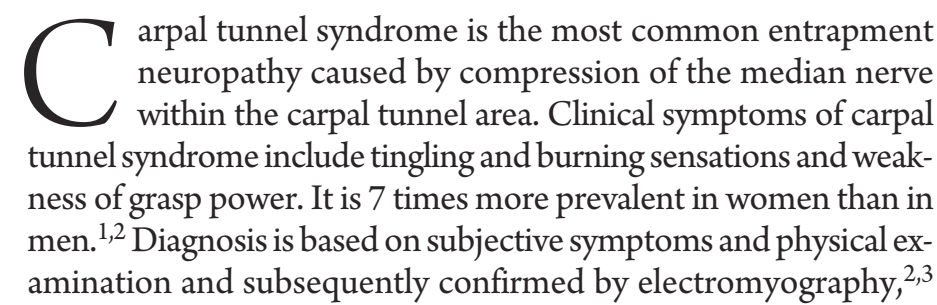


which is a useful diagnostic modality for differentiating carpal tunnel syndrome from other peripheral neuropathies whose symptoms mimic those of carpal tunnel syndrome. ${ }^{4,5}$

However, electromyography is both invasive and painful. Magnetic resonance imaging and sonography are other alternative modalities that assist in the diagnosis of carpal tunnel syndrome. ${ }^{6}$ Because of its reduced costs and feasibility in comparing sides, sonography has recently been more popular. ${ }^{6-8}$ It can depict soft tissue lesions and easily differentiates between various causes of carpal tunnel syndrome, including ganglion cysts, neurogenic tumors, and tenosynovitis. It is also used to detect a bifid median nerve and persistent median artery. ${ }^{4,5}$

Currently, to diagnose carpal tunnel syndrome using sonography, many researchers propose using measurements of the cross-sectional area of the median nerve or obtaining either the flattening or swelling ratio., ${ }^{3,9,10}$ The flattening ratio is obtained by measuring the thickness and width of the median nerve at level of the carpal inlet, whereas the swelling ratio is obtained by dividing the crosssectional area of the median nerve proximal to the flexor retinaculum by the cross-sectional area of the same nerve at the distal end of the flexor retinaculum. ${ }^{3,9,10}$ However, there is no definite consensus on the normal values and ranges of these sonographic indices. ${ }^{11}$

In a comparative cadaveric study, sonography was proven to accurately measure the cross-sectional area of not only the median nerve but also the carpal tunnel. ${ }^{12}$ Hence, we measured distal and proximal cross-sectional areas of the median nerve and carpal tunnel in healthy adults with relation to sex, height, weight, and body mass index (BMI) and developed the nerve/tunnel index, the ratio of the cross-sectional area of the median nerve to that of the carpal tunnel. We also analyzed the relationship between these factors with the intentions of presenting new standards for diagnosing carpal tunnel syndrome with sonography and testing the inter-rater reliability of median nerve and carpal tunnel area measurement using sonography.

\section{Materials and Methods}

This study was conducted from March 2010 to May 2010. Study participants consisted of 60 healthy adults ( 30 male and 30 female) with no signs or symptoms of carpal tunnel syndrome. Exclusion criteria were previous trauma or history of surgery around the wrist; hereditary or acquired diseases, which can cause peripheral neuropathy; mass lesions in the carpal tunnel area, which can cause median nerve compression, including ganglion cysts, neurogenic tumors, flexor tenosynovitis, and accessory flexor digitorum superficalis; and atypical nerve and vessel shapes, such as a bifid median nerve or persistent median artery. The mean ages \pm SDs of the male and female participants were 25.39 \pm 3.69 and $23.79 \pm 3.22$ years, respectively. The study protocol was approved by the Institutional Review Board. Informed consent was obtained from all participants before proceeding with the study. The investigation was governed by ethical principles for experimentation in humans established by the Declaration of Helsinki.

A physiatrist with at least 3 years of clinical experience performed history taking to exclude median nerve injury caused by previous trauma to the wrist and to check for typical symptoms, such as paresthesia and numbness from the thumb to the lateral fourth digit and worsening of symptoms at night or after repetitive activities of the hand and wrist. Physical examinations such as the Phalen test and Tinel sign test were done. The right median nerve was examined with electrodiagnostic equipment (Medelec Synergy; Oxford Instruments Medical, Old Woking, Surrey, England) to obtain the conduction velocity and the amplitude and latency of the motor and sensory nerves. These values were analyzed to exclude the presence of carpal tunnel syndrome. A radiologist with at least 3 years of experience in musculoskeletal sonography performed the sonographic examinations and measured the proximal and distal cross-sectional areas of both the median nerve and carpal tunnel using a 5- to $12-\mathrm{MHz}$ linear array transducer (iU22; Phillips Healthcare, Bothell, WA). The participant was asked to lie supine with the forearm supinated and a shallow pillow underneath the wrist during the sonographic examination.

Proximal and distal cross-sectional areas of the median nerve and carpal tunnel were measured at the scaphoidpisiform and trapezium-hamate levels, respectively. The carpal tunnel's superior and inferior boundaries were the flexor tendon sheath and flexor retinaculum, respectively (Figures 1 and 2). Cross-sectional areas of both the proximal and distal median nerve and carpal tunnel were calculated directly by continuous tracing of the boundaries of the nerve and carpal tunnel along the echogenic boundary.

The proximal and distal nerve/tunnel indices were obtained by calculating the ratio between the proximal or distal cross-sectional area of the median nerve to that of the carpal tunnel and multiplying the value by 100: eg, proximal nerve/tunnel index $=$ (proximal median nerve crosssectional area)/proximal carpal tunnel cross-sectional area) $\times 100$. The inter-rater reliability of the sonographic examination was established by conducting assessments separately by both a radiologist and a physiatrist in 30 participants. 


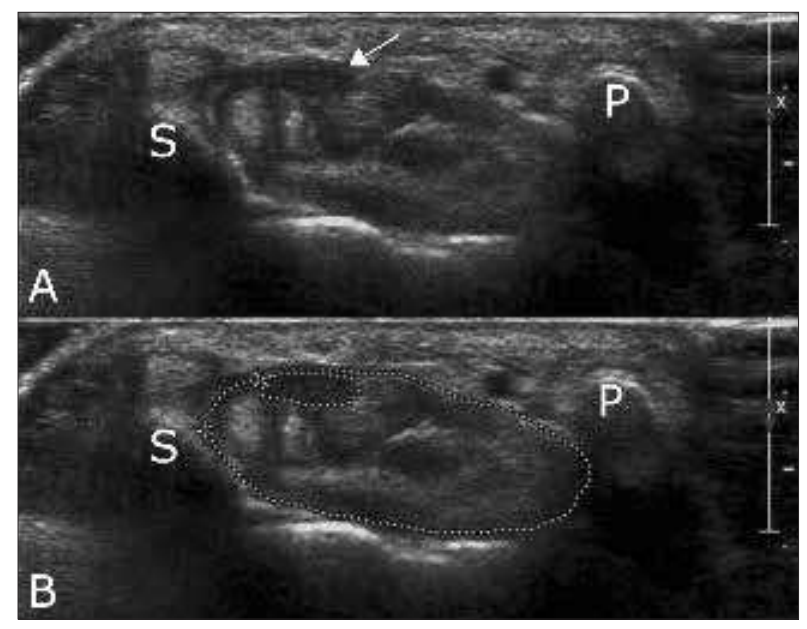

Figure 1. Cross-sectional areas of the median nerve and carpal tunnel at the scaphoid-pisiform level. A, Proximal carpal tunnel, median nerve (arrow), and flexor tendon groups shown between the pisiform (P) and scaphoid (S). B, Dot-outlined proximal median nerve and carpal tunnel.

Data were analyzed statistically using the SPSS version 14.0 statistical package for Windows (Korean version; SPSS Inc, Chicago, IL). Correlation analyses were performed to determine relationships between body indices such as height and weight with sonographic measurement of the proximal and distal cross-sectional areas of the median nerve and carpal tunnel. Independent $t$ tests between male and female participants were performed for age, height, weight, BMI, and sonographic measurements.

Figure 2. Cross-sectional areas of the median nerve and carpal tunnel at the trapezium-hook of the hamate level. A. Proximal carpal tunnel, median nerve (arrow), and flexor tendon groups shown between the trapezium ( $\mathrm{T}$ ) and hook of the hamate $(\mathrm{H})$. B, Dot-outlined distal median nerve and carpal tunnel.

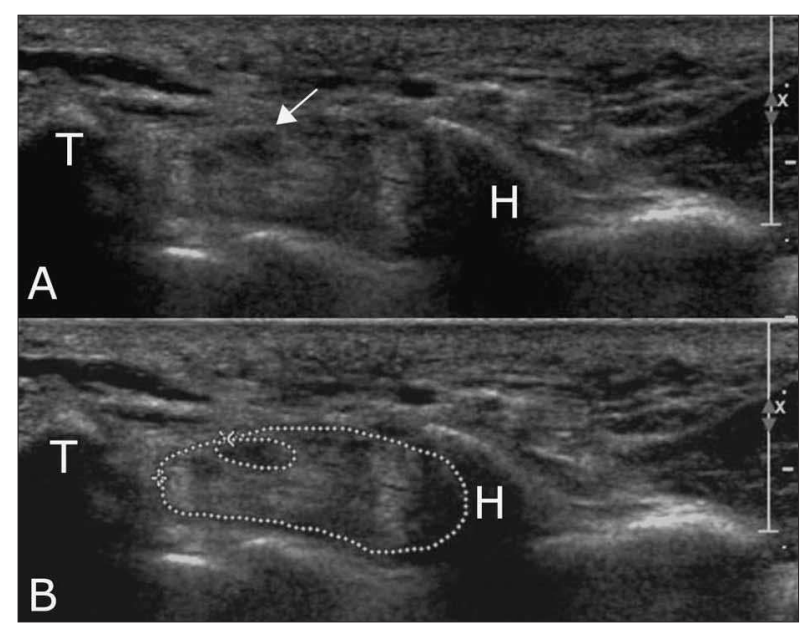

Table 1. Patient Characteristics

\begin{tabular}{lrrl}
\hline Characteristic & \multicolumn{1}{c}{ Male } & \multicolumn{1}{c}{ Female } & $\boldsymbol{P}$ \\
\hline Age, y & $25.39 \pm 3.69$ & $23.79 \pm 3.22$ & .07 \\
Height, cm & $173.90 \pm 4.45$ & $161.93 \pm 4.38$ & .001 \\
Weight, $\mathrm{kg}$ & $71.20 \pm 9.94$ & $52.80 \pm 6.33$ & .001 \\
BMl, kg/m² & $23.61 \pm 2.79$ & $20.10 \pm 1.97$ & .001 \\
\hline
\end{tabular}

Data are mean \pm SD. BMI indicates body mass index.

Comparisons of the proximal and distal cross-sectional areas of the median nerve with the carpal tunnel area and nerve/tunnel index were performed by a paired $t$ test. $P<$ .05 was considered significant for every comparison.

\section{Results}

\section{General Characteristics and Electromyographic Findings}

There was no statistically significant difference in age between the sexes $(P=.07)$, but the height, body weight, and BMI of the male participants were greater than those of the female participants. The mean height, body weight, and BMI of all participants were $167.91 \pm 7.45 \mathrm{~cm}, 62.00 \pm$ $12.42 \mathrm{~kg}$, and $21.85 \pm 2.97 \mathrm{~kg} / \mathrm{m}^{2}$, respectively (Table 1 ). There were no abnormal electromyographic findings in the right median nerve (Table 2), and no participants had sonographic findings indicating the presence of a bifid median nerve or persistent median artery.

\section{Differences Between Sexes in the Proximal and Distal Cross-sectional areas of the Median Nerve and Carpal Tunnel and Proximal and Distal Nerve/Tunnel Indices There were statistically significant differences in the prox- imal and distal cross-sectional areas of the median nerve and carpal tunnel between the male and female partici- pants. However, statistically significant differences in the proximal and distal nerve/tunnel indices were not ob- served between the sexes (Table 3).}

Table 2. Electromyographic Findings

\begin{tabular}{lc}
\hline Electrodiagnostic Index & Value \\
\hline Nerve conduction velocity, m/s & $61.73 \pm 4.23(52.30-73.30)$ \\
Motor amplitude, mV & $17.89 \pm 4.16(8.30-27.70)$ \\
Motor latency, ms & $2.59 \pm 0.27(2.00-3.45)$ \\
Sensory amplitude, mV & $69.71 \pm 19.71(42.00-114.00)$ \\
Sensory latency, ms & $2.29 \pm 0.24(1.85-2.95)$ \\
\hline
\end{tabular}

Data are mean \pm SD (range). 
Table 3. Comparison of Proximal and Distal Cross-sectional Areas of the Median Nerve and Carpal Tunnel and Proximal and Distal Nerve/Tunnel Indices by Sex

\begin{tabular}{lccc}
\hline Sonographic Index & Male & Female & $P$ \\
\hline Proximal CSA of median nerve, $\mathrm{mm}^{2}$ & $10.48 \pm 3.21$ & $8.81 \pm 3.21$ & .023 \\
Proximal CSA of carpal tunnel, $\mathrm{mm}^{2}$ & $182.50 \pm 21.15$ & $151.23 \pm 21.14$ & .001 \\
Proximal nerve/tunnel index, \% & $5.80 \pm 1.72$ & $5.91 \pm 1.63$ & .791 \\
Distal CSA of median nerve, $\mathrm{mm}^{2}$ & $9.99 \pm 3.42$ & $8.46 \pm 1.84$ & .037 \\
Distal CSA of carpal tunnel, $\mathrm{mm}^{2}$ & $138.90 \pm 20.95$ & $121.50 \pm 18.99$ & .001 \\
Distal nerve/tunnel index, \% & $7.15 \pm 2.00$ & $7.01 \pm 1.38$ & .755 \\
\hline
\end{tabular}

Data are mean \pm SD. CSA indicates cross-sectional area.

\section{Relationships Between Body Indices and Sonographic Findings}

Statistically significant relationships were observed between the height, weight, and BMI and the proximal crosssectional area of the median nerve with weak positive associations. However, the distal area of the median nerve was only related to height with a weak positive association, and the proximal and distal nerve/tunnel indices were not significantly related to the body indices. In contrast, the proximal and distal cross-sectional areas of the carpal tunnel were influenced by the body indices with a relatively strong positive correlation (Table 4).

\section{Comparisons of the Proximal to Distal Cross-sectional area of the Median Nerve, Proximal to Distal Cross- sectional area of the Carpal Tunnel, and Proximal to Distal Nerve/Tunnel Index}

There were significant differences between distal and proximal measurements. The proximal cross-sectional areas of the median nerve and carpal tunnel were wider than the respective distal areas. However, the distal nerve/tunnel index was significantly higher than the proximal index (Table 5).

Table 4. Pearson Correlation Coefficients Between Sonographic and Body Indices

\begin{tabular}{lccl}
\hline Sonographic Index & Height & Weight & BMI \\
\hline Proximal CSA of median nerve & $0.300^{\mathrm{a}}$ & $0.357^{\mathrm{b}}$ & $0.357^{\mathrm{b}}$ \\
Proximal CSA of carpal tunnel & $0.594^{\mathrm{b}}$ & $0.594^{\mathrm{b}}$ & $0.470^{\mathrm{b}}$ \\
Proximal nerve/tunnel index & -0.061 & 0.017 & 0.06 \\
Distal CSA of median nerve & $0.275^{\mathrm{a}}$ & 0.228 & 0.229 \\
Distal CSA of carpal tunnel & $0.531^{\mathrm{b}}$ & $0.447^{\mathrm{b}}$ & $0.383^{\mathrm{b}}$ \\
Distal nerve/tunnel index & -0.033 & -0.35 & 0.003 \\
\hline
\end{tabular}

BMI indicates body mass index and CSA, cross-sectional area. aSignificant at $P<.05$ ( 2 tailed).

bSignificant at $P<.01$ ( 2 tailed).

\section{Inter-rater Reliability}

The intraclass correlation coefficients were $0.84,0.76,0.81$, and 0.74 for the proximal median nerve, proximal carpal tunnel, distal median nerve, and distal carpal tunnel crosssectional areas, respectively.

\section{Discussion}

There have been many previous reports of the use of sonography for diagnosing carpal tunnel syndrome. . $3,30,13,14$ Previously studied diagnostic standards described measuring the cross-sectional area of the median nerve and calculating the flattening ratio using sonography. ${ }^{7,8,10,11}$ Most researchers have agreed that the cross-sectional area of the median nerve in carpal tunnel syndrome is larger than that in the unaffected population. However, normal ranges and cutoff values for the median nerve cross-sectional area varied among researchers. ${ }^{6,15}$ Therefore, a lack of consensus remains regarding the most appropriate median nerve cross-sectional area threshold in establishing the diagnosis of carpal tunnel syndrome. ${ }^{16}$ Buchberger et $\mathrm{al}^{10,13}$ documented that the cross-sectional area of the median nerve was abnormal if it was greater than $6 \pm 10 \mathrm{~mm}^{2}$ at the distal radioulnar joint, $6 \pm 11 \mathrm{~mm}^{2}$ at the hamate, and $10 \mathrm{~mm}^{2}$ at the capitate. However, Duncan et $\mathrm{al}^{4}$ stated that the area of the median nerve was abnormal if it was greater than 9 $\mathrm{mm}^{2}$. According to the study by Park et al, ${ }^{7}$ which included 76 healthy participants ( 35 male and 41 female), when comparing the sexes, the male cross-sectional areas of the median nerve measured at the radioulnar joint, pisiform level, and hamate level were greater than the female areas, with mean areas of $6.44 \pm 0.02,6.34 \pm 0.02$, and $6.31 \pm 0.02$ $\mathrm{mm}^{2}$ at the proximal radioulnar joint, capitate level, and hamate level, respectively. These values were smaller compared with other studies. They deduced that the differences in height and weight between Asian and white populations accounted for the results. ${ }^{7}$ 
According to our results and previous reports, the cross-sectional area of the median nerve is variable according to different measurement standards used by different researchers. In addition, our study shows that the cross-sectional area of the median nerve is correlated with body indices, albeit with a weak positive correlation coefficient. In accordance with the greater prevalence of carpal tunnel syndrome in women, our results showed different median nerve areas in the male and female participants. Therefore, it is difficult to present normal reference values of the cross-sectional area of the median nerve without considering sex and body indices. The absence of an agreed-on standard value makes the cross-sectional area of the median nerve a difficult parameter for diagnosing carpal tunnel syndrome. However, because the proximal and distal nerve/tunnel indices are not influenced by either sex or body indices (height, weight, body region, and BMI), they may be useful sonographic parameters for diagnosing carpal tunnel syndrome.

Although the mechanism of carpal tunnel syndrome is due to compression of the median nerve within the carpal tunnel, the underlying etiology is often uncertain. ${ }^{6,17}$ However, Buchberger et $\mathrm{al}^{10}$ reported that sonographic findings of retinacular bowing were observed in 18 patients with a diagnosis of carpal tunnel syndrome, and the distance between the most anterior portion of the transverse carpal ligament and the tubercle of the trapezium and hook of the hamate was increased greater than $3.7 \mathrm{~mm}$ on average. Bianchi and Martinoli ${ }^{11}$ documented that most patients with carpal tunnel syndrome had concomitant finger flexor tenosynovitis, which may often lead to increased intracarpal tunnel pressure causing the transverse carpal ligament to become convex. Thus, they suggested that if the vertical distance between the most anterior portion of the transverse carpal ligament and the imaginary line from the tubercle of the trapezium to hook of the hamate is greater than $4 \mathrm{~mm}$, it can be a pathologic sign of secondary carpal tunnel syndrome due to finger flexor tenosynovitis. If carpal tunnel syndrome is a secondary condition resulting from finger flexor tenosynovitis, the cross-sectional areas of both the median nerve and carpal tunnel will increase. Such similar rates of change in cross-sectional areas will cause only a minimal change in the nerve/tunnel index. Therefore, in such a case, the nerve/tunnel index would have limited value in diagnosing carpal tunnel syndrome because of its low sensitivity. Thus, if the nerve/tunnel index in a patient with carpal tunnel syndrome is not substantially different from that of the unaffected population, it would imply that flexor tenosynovitis may be the cause of the carpal tunnel syndrome. Duncan et $\mathrm{al}^{4}$ performed sonography and magnetic resonance imaging in 102 affected hands of 68 patients with carpal tunnel syndrome. They reported that the clear primary cause in most patients with carpal tunnel syndrome was not found. Secondary causes of carpal tunnel syndrome were found in 35\%, and among the secondary causes, 3 patients had a diagnosis of flexor tenosynovitis. According to El Miedany et al, ${ }^{6} 16$ of 60 patients with unilateral carpal tunnel syndrome had a diagnosis of flexor tenosynovitis. Furthermore, in comparing asymptomatic and symptomatic hands, the cross-sectional area of the median nerve and the flattening ratio showed statistical differences, but the flexor retinaculum thickness and anteroposterior dimensions of the carpal tunnel turned showed no statistically significant differences. ${ }^{18}$ Therefore, the nerve/tunnel index will show increased values in idiopathic carpal tunnel syndrome except in cases in which the cause of carpal tunnel syndrome is a benign tumor such as a fibroma, ganglion cyst, or schwannoma.

In our study of healthy adults without carpal tunnel syndrome, the cross-sectional areas of the proximal median nerve and carpal tunnel were greater than those of the distal median nerve and carpal tunnel, and the distal nerve/tunnel index was also larger than the proximal index. These findings indicate that the proportion of the median nerve to the carpal tunnel is relatively larger at the distal than at the proximal portion of the carpal tunnel. Taking the previously mentioned animal studies, sonographic studies, and our study together, the median nerve may be more easily compressed at or around the distal carpal tunnel.

According to previous epidemiologic studies, the incidence of carpal tunnel syndrome is higher in middle-aged women ${ }^{19}$ and correlates with the BMI and weight. ${ }^{20,21}$

Table 5. Comparison of Proximal to Distal Cross-sectional Area of the Median Nerve, Proximal to Distal Cross-sectional Area of the Carpal Tunnel, and Proximal to Distal Nerve/Tunnel Index

\begin{tabular}{lcc}
\hline Comparison & Value & $\boldsymbol{P}$ \\
\hline Proximal vs distal CSA of median nerve, $\mathrm{mm}^{2}$ & $9.64 \pm 2.86$ vs $9.22 \pm 2.83$ & $<.01$ \\
Proximal vs distal CSA of carpal tunnel, $\mathrm{mm}^{2}$ & $166.87 \pm 27.85$ vs $130.20 \pm 21.27$ & $<.01$ \\
Proximal vs distal nerve/tunnel index, $\%$ & $5.85 \pm 1.66$ vs $7.08 \pm 1.71$ & $<.01$ \\
\hline
\end{tabular}

Data are mean \pm SD. CSA indicates cross-sectional area. 
In our study, the results showed that the nerve/tunnel index was unrelated to the BMI and weight. Thus, the BMI and weight should not be considered possible causative factors of carpal tunnel syndrome because the male median nerve cross-sectional area, BMI, and weight are greater than the female values. If they were causative factors, the incidence of carpal tunnel syndrome in men would be higher than that in women.

Many other previous studies used the absolute crosssectional area of the median nerve without considering sex in the diagnosis of carpal tunnel syndrome by sonography. In these cases, the probability of false-positive results in the diagnosis of carpal tunnel syndrome may increase in men, whereas false-negative results may increase in women.

Klauser et $\mathrm{al}^{16}$ proposed a new method of diagnosing carpal tunnel syndrome using the proximal cross-sectional area of the median nerve at the level of the pronator teres. They compared the cross-sectional areas of the median nerve proximally at the pronator teres level and distally at the scaphoid-pisiform level and verified the difference in the areas between the proximal and distal median nerves in healthy participants and patients with carpal tunnel syndrome. However, sex and correlations between sonographic measurements and height, weight, and BMI were not considered.

The creative idea of our study is that we measured cross-sectional areas of not only the median nerve but also the carpal tunnel and introduced a ratio of these values. It was found that there were significant differences in the proximal and distal measurements for the median nerve and carpal tunnel.

The limitations of this study included relatively lower inter-rater reliability for carpal tunnel area dimension measurement than that of the median nerve. This finding may have been related to the examiner's experience in sonographic evaluation and the resolution of the sonographic machine, but it is speculated that the difference was mainly due to the effect of acoustic shadowing caused by the hook of the hamate on measurement of the distal carpal tunnel area. Therefore, the reproducibility of carpal tunnel area dimensions can be dependent on the experience of the examiner and the resolution of the sonographic equipment. In addition, the inter-rater reliability was tested in only 30 of the 60 patients, which also may have been a limitation of this study. Finally, the participants did not constitute a population in which carpal tunnel syndrome occurs most often; they were not obese and ranged in age from 23 to 25 years. In addition, both wrists were not compared, making it impossible to take right- or left-handedness into consideration.
In conclusion, sonographic measurements of the median nerve and carpal tunnel were greater in the male than the female participants, and the proximal and distal crosssectional areas of the carpal tunnel showed strong correlations with weight, height, and BMI. However, the proximal and distal areas of the median nerve were related to the body indices with a relatively weak positive correlation. It is insufficient to diagnose carpal tunnel syndrome by using the absolute cross-sectional area of the median nerve obtained with sonography. Although the reproducibility of carpal tunnel measurement was lower than that of the median nerve, the intraclass correlation coefficients were sufficiently high to allow sonographic measurement of the carpal tunnel cross-sectional area and the nerve/tunnel index to be used for diagnosis of carpal tunnel syndrome.

\section{References}

1. de Krom MC, Knipschild PG, Kester AD, Thijs CT, Boekkooi PF, Spaans F. Carpal tunnel syndrome: prevalence in the general population. J Clin Epidemiol 1992; 45:373-376.

2. Wang LY, Leong CP, Huang YC, Hung JW, Cheung SM, Pong YP. Best diagnostic criterion in high-resolution ultrasonography for carpal tunnel syndrome. Chang Gung Med J 2008; 31:469-476.

3. Wong SM, Griffith JF, Hui AC, Lo SK, Fu M, Wong KS. Carpal tunnel syndrome: diagnostic usefulness of sonography. Radiology 2004; 232:9399 .

4. Duncan I,Sullivan P, Lomas F. Sonography in the diagnosis of carpal tunnel syndrome. AJR Am J Roentgenol 1999; 173:681-684.

5. Pastare D, Therimadasamy AK, Lee E, Wilder-Smith EP. Sonography versus nerve conduction studies in patients referred with a clinical diagnosis of carpal tunnel syndrome. J Clin Ultrasound 2009; 37:389-393.

6. El Miedany YM, Aty SA, Ashour S. Ultrasonography versus nerve conduction study in patients with carpal tunnel syndrome: substantive or complementary tests? Rheumatology (Oxford) 2004; 43:887-895

7. ParkJY, ParkSR, LeeSH, Choi KH. The ultrasonographic findings of the median nerve in the carpal tunnel according to age and sex of normal Korean adults. J Korean Acad Rehabil Med 2008; 32:564-569.

8. Kim MS, Yoon SY, Lee SO, Lee YK. The abnormal ultrasonographic findings of carpal tunnel in carpal tunnel syndrome. J Korean Acad Rehabil Med 2003; 27:735-739.

9. Choi WK, Kang YK, Kim YH, Park EM. Diagnosis of carpal tunnel syndrome by diagnostic ultrasound. J Korean Acad Rehabil Med 2001; 25:134-139.

10. BuchbergerW,JudmaierW, Birbamer G,Lener M, Schmidauer C. Carpal tunnel syndrome: diagnosis with high-resolution sonography. AJR Am J Roentgenol 1992; 159:793-798

11. BianchiS, Martinoli C.Wrist. In: BianchiS, Martinoli C (eds), Ultrasound of the Musculoskeletal System. Heidelberg, Germany: Springer 2007:445467. 
12. Kamolz LP, Schrogendorfer KF, Rab M, Girsch W, Gruber H, Frey M. The precision of ultrasound imaging and its relevance for carpal tunnel syndrome. Surg Radiol Anat 2001; 23:117-121.

13. Buchberger W, Schon G, Strasser K, Jungwirth W. High-resolution ultrasonography of the carpal tunnel. J Ultrasound Med 1991; 10:531-537.

14. Lee D, van Holsbeeck MT, Janevski PK, Ganos DL, Ditmars DM, Darian VB. Diagnosis of carpal tunnel syndrome: ultrasound versus electromyography. Radiol Clin North Am 1999; 37:859-872.

15. Kaymak B, Ozçakar L, Cetin A, Candan Cetin M, Akinci A, Hasçelik Z.A comparison of the benefits of sonography and electrophysiologic measurements as predictors of symptom severity and functional status in patients with carpal tunnel syndrome. Arch Phys Med Rehabil 2008; 89:743-748.

16. Klauser AS, Halpern EJ, De Zordo T, et al. Carpal tunnel syndrome assessment with US: value of additional cross-sectional area measurements of the median nerve in patients versus healthy volunteers. Radiology 2009; 250:171-177.

17. BuchbergerW. Radiologic imaging of the carpal tunnel. EurJ Radiol 1997; 25:112-117.

18. Martinoli C, BianchiS, Gandolfo N, Valle M, SimonettiS, Derchi LE. US of nerve entrapments in osteofibrous tunnels of the upper and lower limbs. Radiographics 2000; 20(special issue):S199-S213.

19. Dumitru D, Zwarts M. Focal peripheral neuropathy. In: Dumitru D, Amato AA, Zwarts M (eds). Electrodiagnostic Medicine. Philadelphia, PA: Hanley \& Belfus Inc; 2002:1058-1061.

20. Kouyoumdjian JA, Morita MD, Rocha PR, Miranda RC, Gouveia GM. Body mass index and carpal tunnel syndrome. Arq Neuropsiquiatr 2000; 58:252-256.

21. Werner RA, Albers JW, Franzblau A, Armstrong TJ. The relationship between body mass index and the diagnosis of carpal tunnel syndrome. Muscle Nerve 1994; 17:632-636. 